\title{
Application of a silver coating on plastic biliary stents to prevent biofilm formation: an experimental study using electron microscopy
}

Authors

Institutions

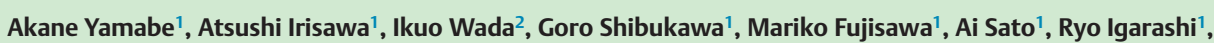
Takumi Maki ${ }^{1}$, Koki Hoshi ${ }^{1}$

${ }^{1}$ Gastroenterology, Aizu Medical Center, Fukushima Medical University, Aizuwakamatsu, Japan

${ }^{2}$ Department of Cell Science, Institute of Biomedical Science, Fukushima Medical University School of Medicine, Fukushima, Japan submitted 21. March 2016 accepted after revision 29. July 2016

\section{Bibliography}

Dol http://dx.doi.org/

10.1055/s-0042-115173

Published online: 14.9.2016

Endoscopy International Open 2016; 04: E1090-E1095

(c) Georg Thieme Verlag KG

Stuttgart · New York

E-ISSN 2196-9736

\section{Corresponding author}

\section{Atsushi Irisawa, MD, PhD}

Department of

Gastroenterology

Aizu Medical Center

Fukushima Medical University

21-2, Maeda, Tanisawa,

Kawahigashi

Aizuwakamatsu, 969-3492

Japan

Fax: +81-242-75-2568

irisawa@fmu.ac.jp
Background and study aims: Biliary stent dysfunction is mainly caused by biliary sludge that forms as a result of bacterial adherence and subsequent biofilm formation on the inner surface of the stent. Silver ions arewell known to have excellent antimicrobial activity against a wide range of microorganisms. In this study, we designed and constructed silver-coated plastic stent (PS) and investigated whether the silver coating prevented bacterial adherence and biofilm formation through the use of electron microscopy.

Material and methods: The polyurethane PS with/ without silver coating were prepared in 6-inch segments. The silver-based antimicrobial agents were electrostatically applied onto the stent surface. The stents were then immersed for 5 weeks in infected human bile juice obtained from a patient with cholangitis, and electron microscopy

\section{Introduction}

$\nabla$

Endoscopic transpapillary stent placement is a common procedure to bypass bile obstruction in patients with malignant or benign diseases of the bile duct. A major adverse effect of this procedure is stent occlusion leading to recurrent jaundice and cholangitis. Two types of stents routinely used in current practice are plastic stents (PS) and self-expanding metal stents (SEMS). Although PS are associated with shorter stent patency than SEMS [1 -4], PS are extremely inexpensive compared to SEMS, and reintervention is easily performed in the event of PS occlusion. As such, if PS had a patency duration comparable to that of SEMS, PS would be recommended as the first choice in the treatment of biliary obstruction.

Stent dysfunction is mainly caused by biliary sludge that forms as a result of bacterial adherence and subsequent biofilm formation on the inner surface of the stent $[5,6]$. Following stent placement across the sphincter of Oddi, low pressure in the bile duct coupled with breaking of the was used to investigate the ability of the modified PS to prevent bacterial adherence and biofilm formation.

Results: The bacterial flora did not change before and after immersion of stents in both the group with and without silver coating. Electron microscopic observation revealed meshwork-like structures around the bacteria, characteristic of biofilm-forming bacteria, in all stents from the control group (6/6, 100\%). On the other hand, a limited number of bacteria were observed in all stents in the silver-coated group, and no apparent biofilm formation was observed $(0 / 6,0 \%)$.

Conclusions: The significance of the findings from our study is the ability of silver-coated PS to prevent biofilm formation on the stent surface, which results in the prevention of stent occlusion.

bacterial barrier facilitates reflux of intestinal contents. This permits easy attachment, colonization and growth of bacteria from the bile on the inner surface of the stent, leading to an ascending bacterial infection $[7,8]$. Because most species of bacteria produce extracellular polysaccharides, the stent surface is often covered with amorphous slime-like coat, collectively called, biofilm. The film can function as a physical barrier to antimicrobials, and renders the colony antimicrobial resistance. The prevention of bacterial infection before the formation of bacterial biofilms on the surface of the stent is thus critical to achieve long-term stent patency.

One area of rapidly increasing interest in the development of new antimicrobials is the use of nanoparticles to coat metals. Specific metal ions such as silver or zinc ions possess antibacterial properties and can be considered as antibacterial substances. Silver ions are well known to have excellent antimicrobial activity against a wide range of microorganisms [9], which is why silver-based nanomaterials are being used for its bactericidal 


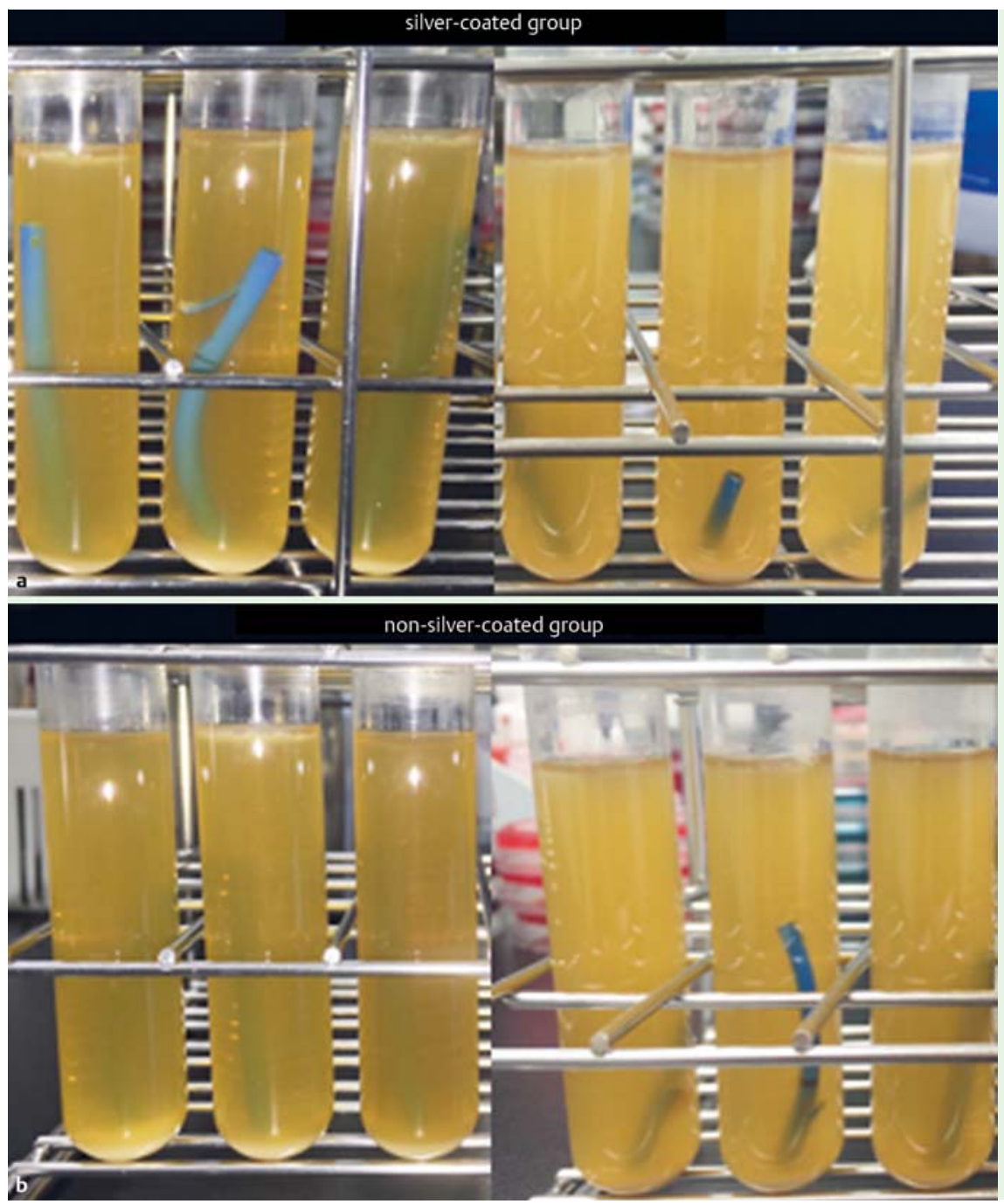

Fig. 1 The color and muddiness of culture media after 5 weeks did not change in either the group with and or without silver coating. a The silvercoated group. $\mathbf{b}$ The non-silver-coated group.

activity $[10,11]$. Interestingly, several studies have shown that silver demonstrates a more potent bactericidal activity against gram-negative bacteria compared to gram-positive bacteria [12, 13]. Consequently, developing a PS product with silver ions could potentially increase stent patency and stability following placement should the ions have sufficient antibacterial activity to reduce bacterial adhesion. Previously, Leung et al. [14] demonstrated that surface modification of polyurethane discs with a coating of silver led to a reduction of adherent bacteria compared to untreated control discs. However, they did not address the issue of bacterial biofilm formation, which is the main factor involved in stent clogging. In this study, we designed and constructed silvercoated PS and investigated whether the silver coating prevented bacterial adherence and biofilm formation through the use of electron microscopy.

\section{Materials and methods \\ $\nabla$}

\section{Study design}

This study was experimental, reviewed and approved by the institutional review board of Fukushima Medical University, and conducted in accordance with the human and ethical principles of research set forth in the Declaration of Helsinki.
The outline for this study is as follows: 1) preparation of polyurethane PS with/without silver coating; 2) immersion of the stents for 5 weeks in infected human bile juice obtained from a patient with cholangitis during an emergent endoscopic retrograde cholangiopancreatography (ERCP) procedure; and 3) investigation with electron microscopy of the ability of these modified PS to prevent bacterial adherence and biofilm formation. Polyurethane plastic biliary stents (Flexima straight type, Boston Scientific Japan, Tokyo, Japan), 6 in 7 Fr and 6 in 8.5 Fr, were used to prepare silver-coated PS. Silver coating was applied to 3 stents of each size to give a total of 6 silver-coated PS and 6 control PS without any silver coating.

Antibiotic-free human bile juice was obtained from a patient with clinically proven obstructive jaundice and acute cholangitis who was undergoing bile drainage during an ERCP procedure (Sept. 7, 2015). The obtained bile juice was immediately divided equally into 12 test tubes. Each PS was then immersed in individual test tubes and cultured in Trypticase Soy Broth (TSB, Difco; Becton, Dickinson and Company, Franklin Lakes, NJ, USA) maintained at $37^{\circ} \mathrm{C}$ for 5 weeks (from Sept. 7, 2015 to Oct. 15, 2015), following which, the PS were removed from the test tubes and subjected to observation under the electron microscope to investigate bacterial adherence and biofilm formation. In addition, microbial validation was performed to analyze the total bacterial flora in the bile juice before and after stent immersion. 

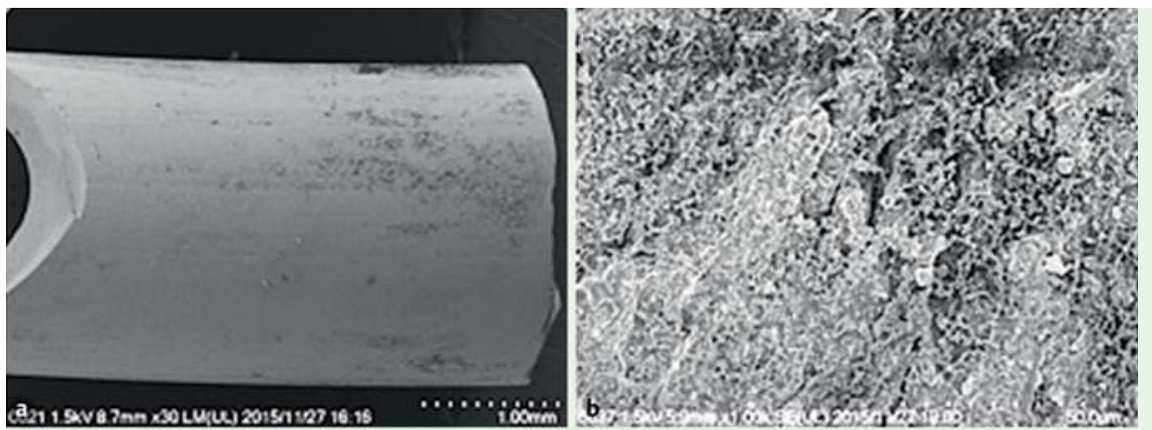

Fig. 2 Electron microscopic analysis of the non-silver-coated plastic biliary stent. Massive bacteria colonies on the polyurethane stent surfaces were developed.
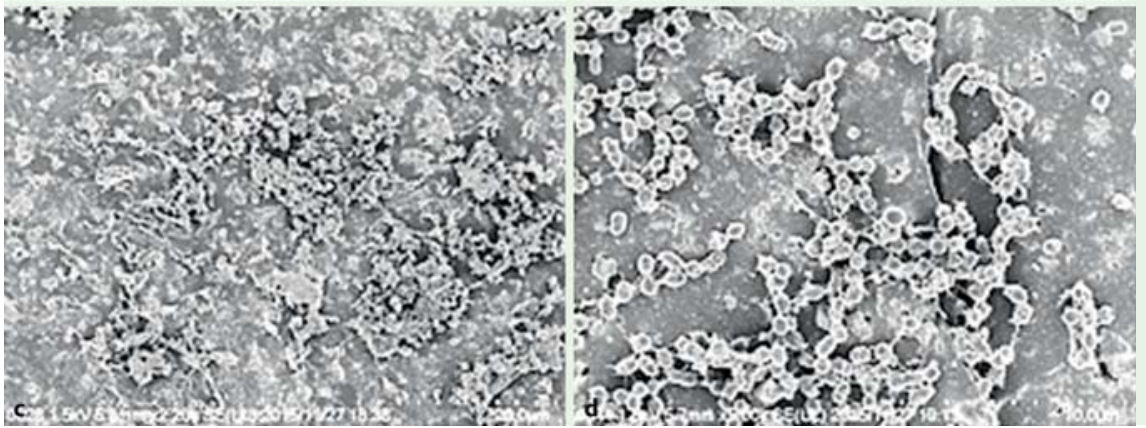

\section{Silver coating technique}

The silver coating technique was provided by Paint Service Corp (Aichi, Japan). Silver-based antimicrobial agents were electrostatically applied onto the wet paint surface. As the paint dried and hardened, the silver-based antimicrobial agents were dispersed uniformly using nanotechnology then partially pulled into the paint surface and firmly fixed. In this study, only the outside of the stent was coated with silver as existing technological limitations render it difficult to uniformly coat the inside of the stent at present. The silver-based antimicrobial particles do not detach easily from the surface of the stent, ensuring antimicrobial efficacy and durability. The costs of the silver coating are about $\$ 5$ per PS.

\section{Scanning electron microscopy}

Samples were fixed with $4 \%$ paraformaldehyde $/ 2.5 \%$ glutaraldehyde in $250 \mathrm{mM}$ HEPES buffer (pH 7.2). After fixing for 30 minutes at room temperature, the samples were washed with phosphate-buffered saline (PBS) 3 times and transferred into $2 \%$ osmium tetroxide in PBS for another 30 minutes. The samples were dehydrated through a graded series of ethanol concentrations, which was eventually replaced with $100 \%$ tert-butyl alcohol then dried in a tert-butyl alcohol freeze-drying apparatus (VFD-21S, Shinku Device, Ibaraki, Japan). After coating with osmium tetroxide in vacuo in a HPC-1SW coater (Shinku Device), images of the specimens' surface were captured with a Hitachi SU8220 scanning electron microscope at an accelerating voltage of $1500 \mathrm{~V}$ according to the manufacturer's protocol.

\section{Results}

$\nabla$

\section{Microbiologic validation}

Analyses of the total bacterial flora in the bile juice before and after immersion of the stents were performed. The following types of bacteria were identified: Escherichia coli, Klebsiella oxytoca, K. pneumoniae ssp. pneumoniae, Enterococcus faecalis, and Enterococcus gallinarum. The color and muddiness of culture media did not change in both groups with and without silver

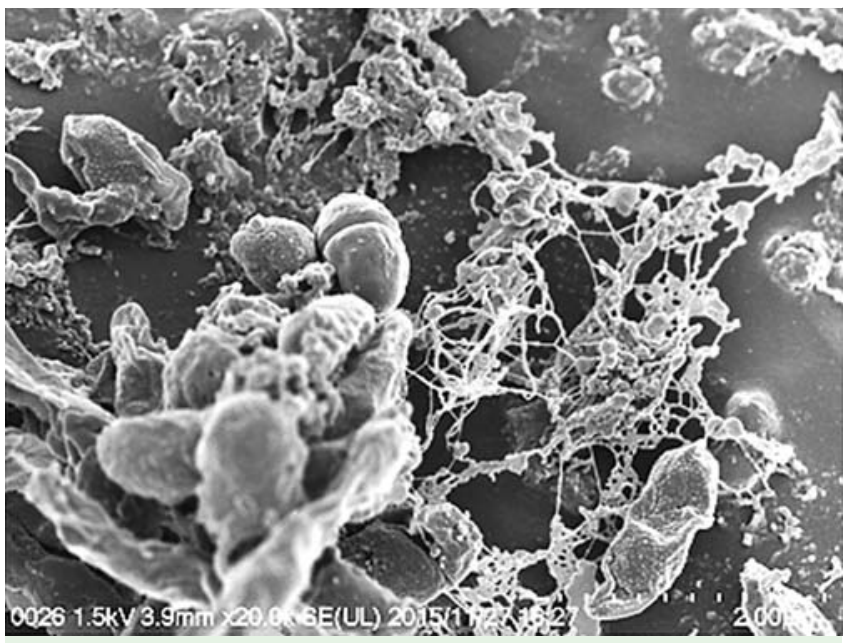

Fig.3 Meshwork-like structures around the bacteria, characteristic of biofilm-forming bacteria, were identified. Under higher magnification, the accumulation of bacteria in the meshwork-like structures by means of connection to each other through fibrous secretions can be seen.

coating. Moreover, the bacterial flora did not change before and after immersion of stents in both groups. Thus, presence of silver coating on the PS per se did not prevent bacterial propagation (० Fig. 1).

\section{Electron microscopic analysis}

Electron microscopic observation revealed meshwork-like structures around the bacteria, characteristic of biofilm-forming bacteria, in all stents from the control group (6/6, 100\%) (० Fig.2). Bacterial cells were often found in such structures interconnected by fibrous materials, and massive bacteria colonies on the polyurethane stent surfaces were developed ( $\mathbf{F i g . 3}$ ). In addition to the mesh-like structures directly associated with bacteria, which appeared to be an initial stage of biofilm formation, flat sludge-like structures were also found in several area of the control stent surface. Bacterial cells were often found at the edge and this appeared to represent the extensively developed biofilm. On 

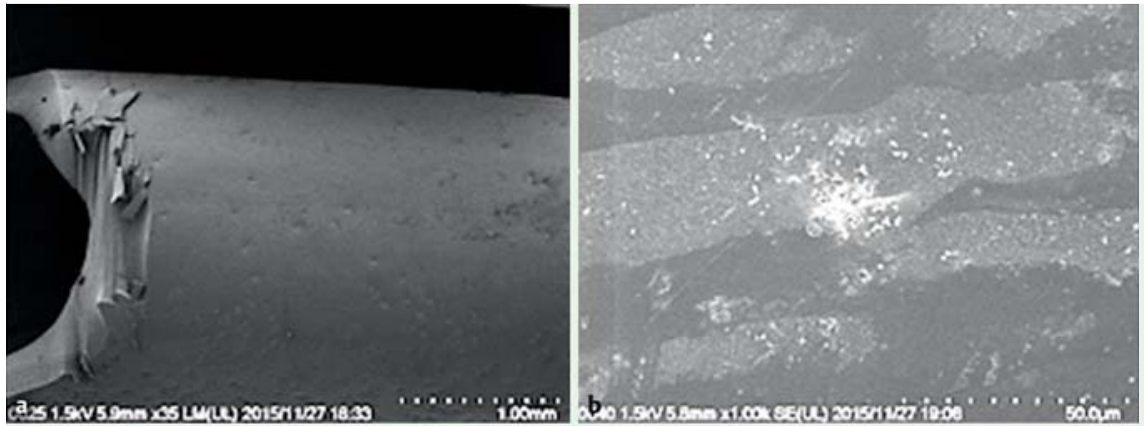

Fig. 4 Electron microscopic analysis of the silvercoated plastic biliary stent. A limited number of bacteria were observed in all stents, and no apparent meshwork-like structures or biofilm formation were identified.
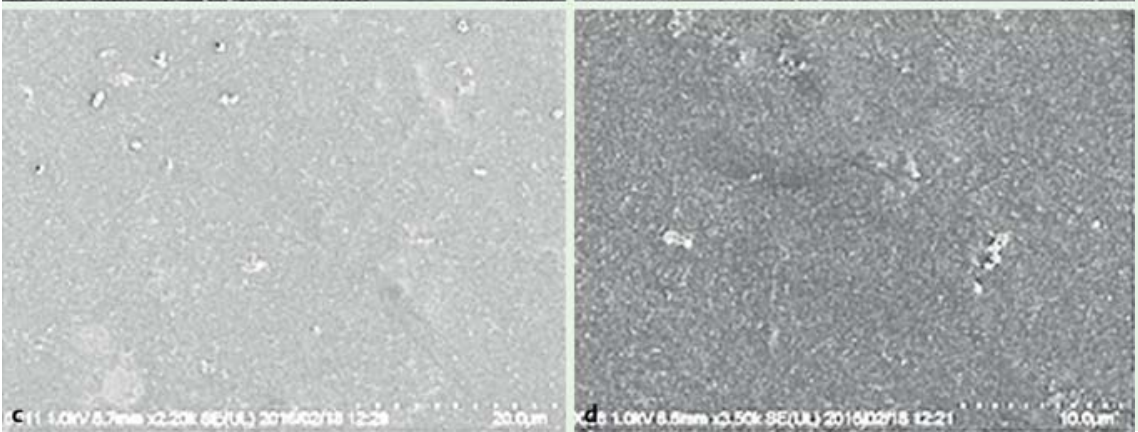

the other hand, limited number of bacteria was observed in all stents in the silver-coated group, and no apparent biofilm formation was identified $(0 / 6,0 \%)(-$ Fig. 4$)$. It should be noted that massive amounts of bacterial colonies were found in the inner stent surfaces in both groups ( $\bullet$ Fig. 5 ).

\section{Discussion}

\section{$\nabla$}

The major limitation of endoscopic stent placement is mainly stent occlusion. It has been reported in the literature that the median patency of PS ranges from 80 days to 126 days $[15,16]$, and stent exchanges are often needed at intervals of approximately 3 months to avoid stent occlusion even if the placed PS remains functional [17]. According to previous studies, stent occlusion is caused by the deposition of biliary sludge, which is composed of cholesterol crystals, calcium bilirubinate and palmitate, bacteria and/or fungi, microbial byproducts, proteins, dietary fibers and glycoproteins [18-20]. In particular, bacterial infection and biofilm formation on the surface of biliary stents is one of the main factors responsible for the failure of stent function. As such, specific modifications made to the surface of PS could potentially allow PS to achieve long-term stent patency. In this study, we investigated the efficacy of an innovative silver-coated plastic stent construct with antibacterial properties.

In vitro studies suggest that bacterial adhesion is the driving force in sludge development $[5,21]$. Moreover, unicellular bacteria in nature prefer to gather to form a surface-attached multi-cellular consortium known as a biofilm rather than living as isolated planktonic cells [22]. Biofilm formation is a common phenomenon such that virtually any material that comes into contact with naturally occurring fluids is rapidly covered with bacteria [23, 24]. Biofilms provide bacteria with various survival and metabolic advantages over the planktonic form. By forming biofilms, bacteria can avail themselves of various advantages including survival in a nutrient-deprived environment, resistance to environmental stresses, resistance to biocides and antibiotics, improved abilities to acquire foreign genes, and many others $[25,26]$.

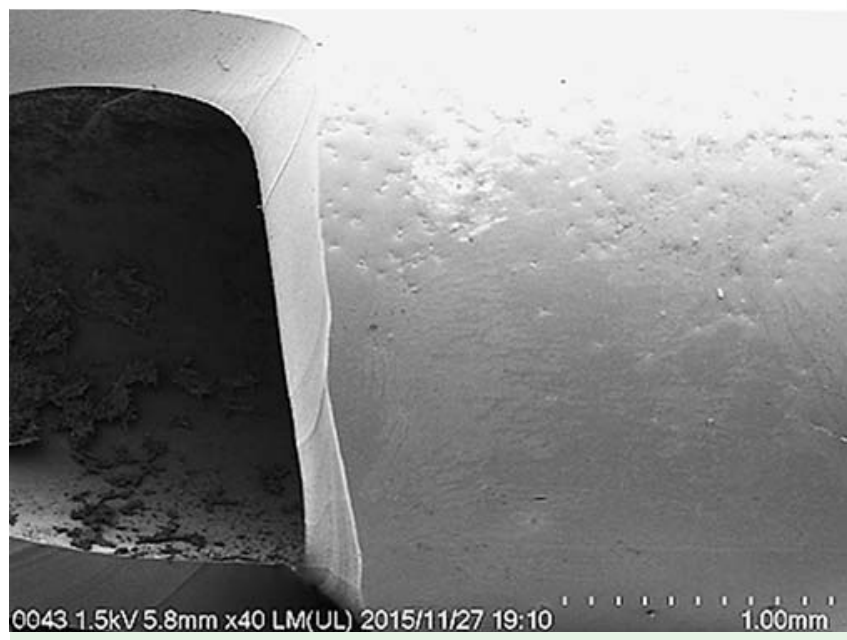

Fig.5 Massive amounts of bacterial colonies were found in the inner stent surfaces in both groups.

One area of rapidly increasing interest in the development of new antimicrobials is the use of nanoparticles coated with metals. Silver, for example, has shown capacity for substantial antimicrobial activity against a diverse range of microorganisms. Silver-based nanomaterials are thus being utilized for their bactericidal activity. Compared to antibiotics, the antimicrobial activity of silver is effective against a wider spectrum of bacteria [27,28]. Complex formation of silver with various components of bacteria, particularly with the respiratory chain, leads to its inhibition, and therefore, the aerobic metabolism of microorganisms is disturbed [29, 30]. Until now, various drugs operating on this concept of antimicrobial activity have been used for therapeutic purposes [3133]. In recent years, novel antimicrobial materials/ catheters effective in preventing the formation of bacterial biofilms have been developed. Importantly, silver ions in particular have proven to be a much more potent inhibitor of biofilm formation than many other antibacterial agents at lower concentrations [34]. Hence, a wide range of antimicrobial catheters based on the bactericidal effect of silver have been developed and marketed suc- 
cessfully. With regard to biliary stents, Leung et al. [5] reported in 1992 that the bacterial count on silver-coated PS surfaces was significantly lower than on the surfaces of PS without any silver coating, suggesting the ability of silver coating to prevent stent occlusion. Wen et al. [35] recently reported the efficacy of a silver-coated stent in a porcine model of bacterial cholangitis. Here, observations under the electron microscope revealed a lower microbial count on the silver-coated stent surface, coupled with a significant reduction in inflammation in the indwelling silver-coated stent group compared to the non-coated stent control group.

Our current finding is essentially consistent to Wen's study. We further showed that mesh-like structures, which were apparently an initial stage of biofilm formation, were observed in all cases on the non-coated stent surfaces, but absent on the silver-coated stent surfaces, despite some bacterial attachment. Our findings strongly suggest that our current method of coating silver on a stent surface is effective in prevention of bacterial meshworklike structures and thus biofilm formation. Because a high density of bacterial colonies was found on the luminal side of the silver-coated stents, it is evident that silver ions are indeed effective in preventing bacterial adhesion and proliferation of adhesive bacteria. While Wen et al. [35] studied the changes in inflammation and bacterial adhesion on stent surfaces over a brief period of 1 day or 7 days after stent placement, our study cultured bacteria on stents immersed in infected bile juice over a longer period of 30 days. Our observation showing near absence of bacterial adhesion on the silver-coated stent surfaces after a longer duration indicates the possibility of achieving of long-term stent patency with silver-coated PS. Interestingly, unlike the observation by Wen et al. [35], which showed that placement of silver-coated stents resulted in significantly reduced inflammatory reaction, we found no changes in bacterial flora in the bile after placement of silver-coated stents. It is thus unlikely that cholangitis that has occurred in vivo can be adequately controlled solely by silver ions dissolved and released into the bile from a silver-coated stent. The significance of the findings from our study is the ability of silver-coated PS in preventing biofilm formation on the stent surface, which results in the prevention of stent occlusion. Nevertheless, our study has several limitations. First, these observations represented in vitro measurements, which may not directly reflect all of the conditions on a plastic stent in vivo. The covering material in vivo would be subjected to a combination of tissue or tumor compression forces and potentially deleterious effects of bile, gastric fluid, pancreatic fluid, and foods we eat. Second, we evaluated a limited number of stents, using only 6 silver-coated PS and 6 control PS without any silver coating. Any long-term benefit in preventing stent blockage will need to be validated by further in vivo studies, more PS and a controlled clinical trial.

In this study, only the outer surface of the stents was coated with silver. Coating both the inner and outer surfaces of stents could lead to the clinical application of biliary stents with long-term patency. Given that PS are much more cost effective than SEMS, this approach to develop silver-coated PS with comparable patency to the SEMS could have considerable cost benefits in the medical economy.

\section{Competing interests: None}

\section{References}

1 Mukai T, Yasuda I, Nakashima M et al. Metallic stents are more efficacious than plastic stents in unresectable malignant hilar biliary strictures: a randomized controlled trial. J Hepatobiliary Pancreat Sci 2013; 20: 214-222

2 Isayama H, Yasuda I, Ryozawa S et al. Results of a Japanese multicenter, randomized trial of endoscopic stenting for non-resectable pancreatic head cancer (JM-test): Covered Wallstent versus DoubleLayer stent. Dig Endosc 2011; 23: 310-315

3 Bernon M, Shaw J, Krige J et al. Malignant biliary obstruction: A prospective randomised trial comparing plastic and metal stents for palliation of symptomatic jaundice. HPB 2011; 13: 139-145

4 Soderlund C, Linder S. Covered metal versus plastic stents for malignant common bile duct stenosis: a prospective, randomized, controlled trial. Gastrointest Endosc 2006; 63: 986-995

5 Leung JW, Ling TK, Kung JL et al. The role of bacteria in the blockage of biliary stents. Gastrointest Endosc 1988; 34: 19-22

6 Speer AG, Cotton PB, Rode J et al. Biliary stent blockage with bacterial biofilm. Ann Intern Med 1988; 108: 546-553

7 Sung JY, Leung JW, Shaffer EA et al. Ascending infection of the biliary tract after surgical sphincterotomy and biliary stenting. J Gastroenterol Hepatol 1992; 7: 240-245

8 Pederson FM, Lassen AT, de Muckadell OBS. Randomized trial of stent placed above and across the sphincter of Oddi in malignant bile duct obstruction. Gastrointest Endosc 1998; 48: 574-579

9 Liau SY, Read DC, Pugh WJ et al. Interaction of silver nitrate with readily identifiable groups: relationship to the antibacterial action of silver ions. Lett Appl Microbiol 1997; 25: 279-283

10 Seo Y, Hwang J, Kim J et al. Antibacterial activity and cytotoxicity of multi-walled carbon nanotubes decorated with silver nanoparticles. Int J Nanomedicine 2014; 9: 4621-4629

11 Neelgund GM, Oki A. Deposition of silver nanoparticles on dendrimer functionalized multiwalled carbon nanotubes: synthesis, characterization and antimicrobial activity. J Nanosci Nanotechnol 2011; 11: $3621-3629$

12 Flores CY, Minan AG, Grillo CA et al. Citrate-capped silver nanoparticles showing good bactericidal effect against both planktonic and sessile bacteria and a low cytotoxicity to osteoblastic cells. ACS Appl Mater Interfaces 2013; 5: 3149-3159

$13 \mathrm{Kim} J S, K u k E, Y u K N$ et al. Antimicrobial effects of silver nanoparticles. Nanomedicine 2007; 3: 95-101

14 Leung JW, Lau GT, Sung JJ et al. Decreased bacterial adherence to silvercoated stent material: an in vitro study. Gastrointest Endosc 1992; 38: $338-340$

15 van Berkel AM, Boland C, Redekop WK et al. A prospective randomized trial of Teflon versus polyethylene stents for distal malignant biliary obstruction. Endoscopy 1998; 30: 681-686

16 Davids PH, Groen AK, Rauws EA et al. Randomised trial of self-expanding metal stents versus polyethylene stents for distal malignant biliary obstruction. Lancet 1992; 340: 1488-1492

17 Dumonceau JM, Tringali A, Blero D et al. Biliary stenting: indications, choice of stents and results: European Society of Gastrointestinal Endoscopy (ESGE) clinical guideline. Endoscopy 2012; 44: 277-298

18 Dowidar N, Kolmos HJ, Lyon $\mathrm{H}$ et al. Clogging of biliary endoprostheses. A morphologic and bacteriologic study. Scand J Gastroentero 1991; 26: $1137-1144$

19 McAllister EW, Carey LC, Brady PG et al. The role of polymeric surface smoothness of biliary stents in bacterial adherence, biofilm deposition, and stent occlusion. Gastrointest Endosc 1993; 39: 422 - 425

20 Weickert $U$, Venzke T, Konig J et al. Why do bilioduodenal plastic stents become occluded? A clinical and pathological investigation on 100 consecutive patients. Endoscopy 2001; 33: 786-790

21 Dowidar N, Kolmos HJ, Matzen P. Experimental clogging of biliary endoprostheses. Role of bacteria, endoprosthesis material, and design. Scand J Gastroenterol 1992; 27: 77-80

22 Ahmad SAI, Bari SMN, Mohiuddin M. Biofilm: multicellular living of the unicellular bacteria. International Journal of Biosciences 2012; 2: 59 71

23 Costerton JW, Cheng KJ, Geesey GG et al. Bacterial biofilms in nature and disease. Annu Rev Microbiol 1987; 41: 435 - 464

24 Costerton JW, Lewandowski Z, DeBeer D et al. Biofilms, the customized microniche. J Bacteriol 1994; 176: 2137-2142

25 Davey ME, O'Toole GA. Microbial biofilms: from ecology to molecular genetics. Microbiol Mol Biol Rev 2000; 64: 847-867 
26 Watnick P, Kolter R. Biofilm, city of microbes. J Bacteriol 2000; 182: $2675-2679$

27 Kumar R, Munstedt H. Silver ion release from antimicrobial polyamide/ silver composites. Biomaterials 2005; 26: 2081 -2088

28 Lee D, Cohen RE, Rubner MF. Antibacterial properties of Ag nanoparticle loaded multilayers and formation of magnetically directed antibacterial microparticles. Langmuir 2005; 21: 9651 -9659

29 Schierholz JM, Lucas LJ, Rump A et al. Efficacy of silver-coated medical devices. J Hosp Infect 1998; 40: 257-262

30 Morones JR, Elechiguerra JL, Camacho A et al. The bactericidal effect of silver nanoparticles. Nanotechnology 2005; 16: 2346-2353
31 Russell $A D$, Hugo WB. Antimicrobial activity and action of silver. Prog Med Chem 1994; 31: 351-370

32 Carsin H, Wassermann D, Pannier $M$ et al. A silver sulphadiazine-impregnated lipidocolloid wound dressing to treat second-degree burns. J Wound Care 2004; 13: 145-148

33 Wright JB, Lam $K$, Hansen $D$ et al. Efficacy of topical silver against fungal burn wound pathogens. Am J Infect Control 1999; 27: 344-350

34 Kumon H. Pathogenesis and management of bacterial biofilms in the urinary tract. J Infect Chemother 1996; 2: 18-28

35 Wen W, Ma LM, He W et al. Silver-nanoparticle-coated biliary stent inhibits bacterial adhesion in bacterial cholangitis in swine. Hepatobiliary Pancreat Dis Int 2016; 15: 87-92 